\title{
Global asymptotic stability of nonautonomous Cohen-Grossberg neural network models with infinite delays
}

\author{
Salete Esteves ${ }^{\ddagger}$ and José J. Oliveira* \\ (ł) Escola Superior de Comunicação, Administração e Turismo \\ Instituto Politécnico de Bragança \\ Rua João Maria Sarmento Pimentel, Apartado 128, 5370-326 Mirandela, Portugal \\ e-mail: saleteesteves@ipb.pt \\ (*) Centro de Matemática (CMAT), Departamento de Matemática e Aplicações, \\ Universidade do Minho, Campus de Gualtar, 4710-057 Braga, Portugal \\ e-mail: jjoliveira@math.uminho.pt
}

\begin{abstract}
For a general Cohen-Grossberg neural network model with potentially unbounded time-varying coefficients and infinite distributed delays, we give sufficient conditions for its global asymptotic stability. The model studied is general enough to include, as subclass, the most of famous neural network models such as Cohen-Grossberg, Hopfield, and bidirectional associative memory. Contrary to usual in the literature, in the proofs we do not use Lyapunov functionals. As illustrated, the results are applied to several concrete models studied in the literature and a comparison of results shows that our results give new global stability criteria for several neural network models and improve some earlier publications.
\end{abstract}

Keywords: Cohen-Grossberg neural networks, unbounded time-varying coefficients, unbounded distributed delays, global asymptotic stability.

Mathematics Subject Classification: 34K20, 34K25, 34K60, 92B20.

\section{Introduction}

The Cohen-Grossberg neural network models, first proposed and studied by Cohen and Grossberg [6] in 1983, have been the subject of an active research due to their extensive applications in various engineering and scientific areas such as neural-biology, population biology, and computing technology. The neural network model in [6] can be described by the following system of ordinary differential equations

$$
x_{i}^{\prime}(t)=-\rho_{i}\left(x_{i}(t)\right)\left[b_{i}\left(x_{i}(t)\right)-\sum_{j=1}^{n} c_{i j} f_{j}\left(x_{j}(t)\right)+I_{i}\right], t \geq 0, i=1, \ldots, n,
$$

which includes, as a special case, the Hopfield neural network model

$$
x_{i}^{\prime}(t)=-b_{i}\left(x_{i}(t)\right)+\sum_{j=1}^{n} c_{i j} f_{j}\left(x_{j}(t)\right)+I_{i}, \quad t \geq 0, i=1, \ldots, n,
$$

studied by Hopfield [12] in 1984. In 1988, Kosko [15] presented a kind of neural network models, known as bidirectional associative memory (BAM), described by

$$
\left\{\begin{array}{l}
x_{i}^{\prime}(t)=-x_{i}(t)+\sum_{j=1}^{n} a_{i j} f_{j}\left(y_{j}(t)\right)+I_{i} \\
y_{i}^{\prime}(t)=-y_{i}(t)+\sum_{j=1}^{n} b_{i j} g_{j}\left(x_{j}(t)\right)+J_{i}
\end{array} \quad t \geq 0, i=1, \ldots, n .\right.
$$


The following generalization of the Cohen-Grossberg model (1.1),

$$
x_{i}^{\prime}(t)=-\rho_{i}\left(x_{i}(t)\right)\left[b_{i}\left(x_{i}(t)\right)+\sum_{j=1}^{n} f_{i j}\left(x_{j}(t)\right)\right], t \geq 0, i=1, \ldots, n,
$$

introduced in [19], includes most of neural network models as special cases, namely (1.1), (1.2), and (1.3).

In order to be more realistic, differential equations describing neural networks should incorporate time delays to take into account the synaptic transmission time among neurons, or, in artificial neural networks, the communication time among amplifiers. In 1989, Marcus and Westervelt [18] introduced for the first time a discrete delay in the Hopfield model (1.2), and they observed that the delay can destabilize the system. In fact, the delays can affect the dynamic behavior of neural network models [1] and, for this reason, stability of delayed neural networks has been investigated extensively ([2], [3], [7], [8], [13], [14], [16], [19], [20], [23], [25], and the references therein). Another relevant fact to take into account is that the neuron charging time, the interconnection weights, and the external inputs often change as time proceeds. Thus, the neural network models with temporal structure of neural activities should be introduced and investigated (see [5], [23]).

For neural network models with time-varying coefficients, many authors assume the existence of an equilibrium point and study its global exponential stability (see [17], [21], [26]). Other authors study the global exponential stability of the models but, in these cases, they assume that the coefficients are bounded functions (see [4], [23], [24], and the references therein). Studies about global asymptotic stability of nonautonomous neural network models are few and the authors always assume bounded time-varying coefficients (see [14], [16], [22], [25]). This paper aims at the global asymptotic stability of a general nonautonomous Cohen-Grossberg neural network model without assuming the existence of an equilibrium point or bounded time-varying coefficients.

Moreover, the models studied here have infinite delays and, when we are dealing with functional differential equations with infinite delays, the choice of an admissible Banach phase space requires special attention in order to have well-posedness of the initial value problem and standard results on existence, uniqueness, and continuation of solutions (see [9], [10], [11]). We note that many papers, dealing with neural networks with unbounded delays, do not provide an explicit phase space, much less the problem of such a suitable choice, and it is known that the choice of the phase space is not the same when we are studying the global exponential stability or we are studying the global asymptotic stability [8].

After the introduction, the present paper is divided into three sections. Section 2 is a preliminary section, where some notation and definition are introduced and the phase space for the models is presented. In Section 3, we present the results on global asymptotic stability of a general nonautonomous Cohen-Grossberg neural network model with infinite delays, which includes most of neural network models. It is important to note that most studies use a type of Lyapunov functional to obtain results on global attractivity (see [4], [5], [14], [16], [24], [25]). Instead, here we use the techniques described in the work of Oliveira [20], rather than a Lyapunov functional approach. In fact, we consider a Cohen-Grossberg model in general setting for which, assuming the existence of instantaneous negative feedbacks which dominate the delay effect, we prove that all solutions are defined on $\mathbb{R}$ and then we prove the global asymptotic stability of the model. Finally, in Section 4, we illustrate the results with well-known neural network examples and we compare our results with the literature, presenting new stability criteria.

\section{Notations and definitions}

We denote by $B C=B C\left((-\infty, 0] ; \mathbb{R}^{n}\right)$ the space of bounded and continuous functions, $\phi:(-\infty, 0] \rightarrow$ $\mathbb{R}^{n}$, equipped with the norm $\|\phi\|=\sup _{s \leq 0}|\phi(s)|$, where $|\cdot|$ is the maximum norm in $\mathbb{R}^{n}$, i.e. $|x|=$ $\max \left\{\left|x_{i}\right|: i=1, \ldots, n\right\}$ for $x=\left(x_{1}, \ldots, x_{n}\right) \in \mathbb{R}^{n}$. For $a \in \mathbb{R}^{n}$, we also use $a$ to denote the constant function $\varphi(s)=a$ in $B C$. A vector $c=\left(c_{1}, \ldots, c_{n}\right) \in \mathbb{R}^{n}$ is said to be positive if $c_{i}>0$ for all $i \in\{1, \ldots, n\}$ and in this case we write $c>0$. 
For an open set $D \subseteq B C$ and $f:[0,+\infty) \times D \rightarrow \mathbb{R}^{n}$ a continuous function, consider the functional differential equation (FDE) given in general setting by

$$
\dot{x}(t)=f\left(t, x_{t}\right), \quad t \geq 0
$$

where, as usual, $x_{t}$ denotes the function $x_{t}:(-\infty, 0] \rightarrow \mathbb{R}^{n}$ defined by $x_{t}(s)=x(t+s)$ for $s \leq 0$.

It is well-known that the Banach space $B C$ is not an admissible phase space for (2.1), in the sense of [9], thus the standard existence, uniqueness, continuous dependence type results are not available. Instead of $B C$, we consider the admissible Banach space

$$
U C_{g}=\left\{\phi \in C\left((-\infty, 0] ; \mathbb{R}^{n}\right): \sup _{s \leq 0} \frac{|\phi(s)|}{g(s)}<\infty, \frac{\phi(s)}{g(s)} \text { is uniformly continuous on }(-\infty, 0]\right\},
$$

equipped with the norm $\|\phi\|_{g}=\sup _{s \leq 0} \frac{|\phi(s)|}{g(s)}$, where $g:(-\infty, 0] \rightarrow[1, \infty)$ is a function satisfying:

(g1) $g$ is a non-increasing continuous function and $g(0)=1$;

(g2) $\lim _{u \rightarrow 0^{-}} \frac{g(s+u)}{g(s)}=1$ uniformly on $(-\infty, 0]$;

(g3) $g(s) \rightarrow+\infty$ as $s \rightarrow-\infty$.

For example, the function $g(s)=e^{-\beta s}$ with $\beta>0$ satisfies (g1)-(g3).

As $B C \subseteq U C_{g}$, then $B C$ is a subspace of $U C_{g}$, and we denote by $B C_{g}$ the space $B C$ with the norm $\|\cdot\|_{g}$. When $n=1$, we denote the spaces $U C_{g}$ and $B C$ by $U C_{g}^{1}$ and $B C^{1}$ respectively.

As $U C_{g}$ is an admissible Banach space, we consider the FDE (2.1) in the phase space $U C_{g}$, for a convenient function $g$, and we assume that $f$ has enough smooth properties to ensure the existence and uniqueness of solution for the initial value problem (see [10]). The solution of (2.1) with initial condition $x_{t_{0}}=\varphi$, for $t_{0} \geq 0$ and $\varphi \in U C_{g}$, is denoted by $x\left(t, t_{0}, \varphi\right)$. In view of our applications to neural network systems, we restrict our attention to initial bounded conditions, i.e.,

$$
x_{t_{0}}=\varphi, \quad \text { with } \varphi \in B C,
$$

for some $t_{0} \geq 0$. From [10], if $f$ maps closed bounded subsets of its domain into bounded sets of $\mathbb{R}^{n}$, then the solution of $(2.1)$ and $(2.2)$ is extensible to $(-\infty, a]$, with $a>t_{0}$, whenever it is bounded.

Definition 2.1. The system (2.1) is said to be

(i) stable if for any $t_{0}>0, \varepsilon>0$, there is $\delta>0$ such that, for all $\varphi, \widetilde{\varphi} \in B C$,

$$
\|\varphi-\widetilde{\varphi}\|<\delta \Rightarrow\left|x\left(t, t_{0}, \varphi\right)-x\left(t, t_{0}, \widetilde{\varphi}\right)\right|<\varepsilon, \text { for all } t \geq t_{0}
$$

(ii) globally asymptotically stable if it is stable and

$$
\lim _{t \rightarrow+\infty}\left(x\left(t, t_{0}, \varphi\right)-x\left(t, t_{0}, \widetilde{\varphi}\right)\right)=0,
$$

for any $t_{0} \geq 0$ and $\varphi, \widetilde{\varphi} \in B C$.

It should be mentioned that the above definition of stability is the usual one in the literature on neural networks with unbounded delay, but it does not even imply the stability in the phase space $U C_{g}$, i.e., relative to the norm $\|\cdot\|_{g}$.

Finally, a function $\gamma:[a,+\infty) \rightarrow \mathbb{R}, a \in \mathbb{R}$, is said to be eventually monotone if there is $t^{*}>a$ such that $\gamma$ is non-decreasing (or non-increasing) on $\left[t^{*},+\infty\right)$. For a bounded real function $h: U \subseteq \mathbb{R} \rightarrow \mathbb{R}$, we denote $\bar{h}:=\sup _{s \in U} h(s)$ and $\underline{h}:=\inf _{s \in U} h(s)$. An increasing sequence $\left(a_{n}\right)_{n \in \mathbb{N}}$ which converges to $a \in \mathbb{R} \cup\{+\infty\}$ as $n \rightarrow+\infty$ is denoted by $a_{n} \nearrow a$. 


\section{Asymptotic stability}

In the phase space $U C_{g}$, with $g:(-\infty, 0] \rightarrow[1,+\infty)$ being a function satisfying (g1)-(g3), consider the following general nonautonomous Cohen-Grossberg neural network model with distributed delays

$$
x_{i}^{\prime}(t)=-\rho_{i}\left(x_{i}(t)\right)\left[b_{i}\left(t, x_{i}(t)\right)+\sum_{j=1}^{n} f_{i j}\left(t, x_{j, t}\right)\right], t \geq 0, i=1, \ldots, n,
$$

where $\rho_{i}: \mathbb{R} \rightarrow(0,+\infty), f_{i j}:[0,+\infty) \times U C_{g}^{1} \rightarrow \mathbb{R}$, and $b_{i}:[0,+\infty) \times \mathbb{R} \rightarrow \mathbb{R}$ are continuous functions. This model is a generalization of a model introduced in [19] and it is particularly relevant in terms of applications, since it includes different types of neural network models with delays, such as Hopfield, Cohen-Grossberg, and BAM.

For (3.1) the following hypotheses will be considered:

(A1) for each $i \in\{1, \ldots, n\}$, there exist $\bar{\rho}_{i}, \underline{\rho}_{i}>0$ such that

$$
\underline{\rho}_{i} \leq \rho_{i}(u) \leq \bar{\rho}_{i}, \quad \forall u \in \mathbb{R}
$$

(A2) for each $i \in\{1, \ldots, n\}$, there exists a function $\beta_{i}:[0,+\infty) \rightarrow(0,+\infty)$ such that

$$
\frac{b_{i}(t, u)-b_{i}(t, v)}{u-v} \geq \beta_{i}(t), \quad \forall t \geq 0, \forall u, v \in \mathbb{R}, u \neq v
$$

(A3) for each $i, j \in\{1, \ldots, n\}$, there exists a function $l_{i j}:[0,+\infty) \rightarrow[0,+\infty)$ such that

$$
\left|f_{i j}(t, \varphi)-f_{i j}(t, \psi)\right| \leq l_{i j}(t)\|\varphi-\psi\|_{g}, \quad \forall t \geq 0, \forall \varphi, \psi \in B C^{1} ;
$$

(A4) there exists $\alpha>0$ such that, for each $i \in\{1, \ldots, n\}$,

$$
\underline{\rho}_{i} \beta_{i}(t)-\sum_{j=1}^{n} \bar{\rho}_{j} l_{i j}(t)>\alpha, \quad \forall t \geq 0 \text {. }
$$

We remark that the hypothesis (A2) says that, for each $t \geq 0$ and $i \in\{1, \ldots, n\}$, the function $u \mapsto b_{i}(t, u)$ is increasing with a growth rate at least $\beta_{i}(t)$. For example, (A2) is trivially satisfied if $b_{i}(t, u)=\beta_{i}(t) u$ for all $t \geq 0$ and $u \in \mathbb{R}$.

In the following Lemma, we show that the solutions of (3.1), with bounded initial condition, are defined on $\mathbb{R}$.

Lemma 3.1. For (3.1) assume (A1)-(A4). Then, each solution $x(t)=x\left(t, t_{0}, \varphi\right)$ (with $t_{0} \geq 0$ and $\varphi \in B C$ ) of (3.1) is defined on $\mathbb{R}$.

Proof. Let $x(t)=\left(x_{1}(t), \ldots, x_{n}(t)\right)$ be a noncontinuable solution of $(3.1)$ on $(-\infty, a)$, for some $t_{0} \geq 0$ and $a \in\left(t_{0},+\infty\right]$, such that $x_{t_{0}}=\varphi$ with $\varphi \in B C$.

Assume that $a \in\left(t_{0},+\infty\right)$. Then, from the Continuation Theorem (see [10]), $\|x(t)\| \rightarrow+\infty$ as $t \rightarrow$ $a^{-}$and, defining $z(t)=\left(z_{1}(t), \ldots, z_{n}(t)\right):=\left(\bar{\rho}_{1}^{-1}\left|x_{1}(t)\right|, \ldots, \bar{\rho}_{n}^{-1}\left|x_{n}(t)\right|\right)$, there exist $i \in\{1, \ldots, n\}$ and a sequence $\left(t_{k}\right)_{k \in \mathbb{N}}$ such that $t_{k} \nearrow a, z_{i}\left(t_{k}\right) \nearrow+\infty$, and

$$
z_{i}\left(t_{k}\right)=\left\|z_{t_{k}}\right\| \geq\left\|z_{t_{k}}\right\|_{g}>0 \quad \text { and } \quad z_{i}^{\prime}\left(t_{k}\right) \geq 0, \quad \forall k \in \mathbb{N} .
$$


From the hypotheses and (3.3) we conclude that, for each $k \in \mathbb{N}$,

$$
\begin{aligned}
& z_{i}^{\prime}\left(t_{k}\right)=\bar{\rho}_{i}^{-1} \operatorname{sign}\left(x_{i}\left(t_{k}\right)\right) x_{i}^{\prime}\left(t_{k}\right) \\
& =\bar{\rho}_{i}^{-1} \operatorname{sign}\left(x_{i}\left(t_{k}\right)\right)\left[-\rho_{i}\left(x_{i}\left(t_{k}\right)\right)\left(b_{i}\left(t_{k}, x_{i}\left(t_{k}\right)\right)+\sum_{j=1}^{n} f_{i j}\left(t_{k}, x_{j, t_{k}}\right)\right)\right] \\
& =-\bar{\rho}_{i}^{-1} \rho_{i}\left(x_{i}\left(t_{k}\right)\right) \operatorname{sign}\left(x_{i}\left(t_{k}\right)\right)\left[\left(b_{i}\left(t_{k}, x_{i}\left(t_{k}\right)\right)-b_{i}\left(t_{k}, 0\right)\right)+\right. \\
& \left.\sum_{j=1}^{n}\left(f_{i j}\left(t_{k}, x_{j, t_{k}}\right)-f_{i j}\left(t_{k}, 0\right)\right)+\left(b_{i}\left(t_{k}, 0\right)+\sum_{j=1}^{n} f_{i j}\left(t_{k}, 0\right)\right)\right] \\
& \leq-\rho_{i}\left(x_{i}\left(t_{k}\right)\right)\left[\beta_{i}\left(t_{k}\right) \bar{\rho}_{i}^{-1}\left|x_{i}\left(t_{k}\right)\right|-\bar{\rho}_{i}^{-1} \sum_{j=1}^{n} l_{i j}\left(t_{k}\right)\left\|x_{j, t_{k}}\right\|_{g}-\bar{\rho}_{i}^{-1}\left|b_{i}\left(t_{k}, 0\right)+\sum_{j=1}^{n} f_{i j}\left(t_{k}, 0\right)\right|\right] \\
& \leq-\rho_{i}\left(x_{i}\left(t_{k}\right)\right)\left[\beta_{i}\left(t_{k}\right) z_{i}\left(t_{k}\right)-\bar{\rho}_{i}^{-1} \sum_{j=1}^{n} l_{i j}\left(t_{k}\right) \bar{\rho}_{j}\left\|z_{j, t_{k}}\right\|-\bar{\rho}_{i}^{-1}\left|b_{i}\left(t_{k}, 0\right)+\sum_{j=1}^{n} f_{i j}\left(t_{k}, 0\right)\right|\right] \\
& \leq-\rho_{i}\left(x_{i}\left(t_{k}\right)\right)\left[\beta_{i}\left(t_{k}\right) z_{i}\left(t_{k}\right)-\left(\sum_{j=1}^{n} \frac{\bar{\rho}_{j}}{\bar{\rho}_{i}} l_{i j}\left(t_{k}\right)\right)\left\|z_{t_{k}}\right\|-M_{i}\right] \\
& =-\rho_{i}\left(x_{i}\left(t_{k}\right)\right)\left[\left(\beta_{i}\left(t_{k}\right)-\sum_{j=1}^{n} \frac{\bar{\rho}_{j}}{\bar{\rho}_{i}} l_{i j}\left(t_{k}\right)\right) z_{i}\left(t_{k}\right)-M_{i}\right] \leq-\rho_{i}\left(x_{i}\left(t_{k}\right)\right)\left(\frac{\alpha}{\underline{\rho}_{i}} z_{i}\left(t_{k}\right)-M_{i}\right) \text {, }
\end{aligned}
$$

where $M_{i}:=\max _{t \in\left[t_{0}, a\right]} \bar{\rho}_{i}^{-1}\left|b_{i}(t, 0)+\sum_{j=1}^{n} f_{i j}(t, 0)\right|$. As $\alpha>0, \rho_{i}(u) \geq \underline{\rho}_{i}>0$ for all $u \in \mathbb{R}$, and $z_{i}\left(t_{k}\right) \nearrow+\infty$ as $k \rightarrow+\infty$, then, for large $k$, we have $z_{i}^{\prime}\left(t_{k}\right)<0$ which is a contradiction. Thus $a=+\infty$.

For $t_{0}>0$ and $\varphi, \psi \in B C$, consider the solutions $x(t)=x\left(t, t_{0}, \varphi\right)$ and $y(t)=x\left(t, t_{0}, \psi\right)$ of $(3.1)$. As $x(t)$ and $y(t)$ are defined on $\mathbb{R}$, we define, for all $t \geq t_{0}, V(t)=V\left(t, t_{0}, \varphi, \psi\right)=\left(V_{1}(t), \ldots, V_{n}(t)\right)$ by

$$
V_{i}(t):=\operatorname{sign}\left(x_{i}(t)-y_{i}(t)\right) \int_{y_{i}(t)}^{x_{i}(t)} \frac{1}{\rho_{i}(s)} d s, \quad i=1, \ldots, n .
$$

Thus, we have $V(t) \geq 0$ for all $t \geq t_{0}$ and, from (A1), we conclude that $\lim _{t \rightarrow+\infty} V(t)=0$ if and only if $\lim _{t \rightarrow+\infty}(x(t)-y(t))=0$.

For each $i \in\{1, \ldots, n\}$ and $t \geq t_{0}$ such that $V_{i}(t) \neq 0$, we have

$$
\begin{aligned}
V_{i}^{\prime}(t) & =\left(\frac{1}{\rho_{i}\left(x_{i}(t)\right)} x_{i}^{\prime}(t)-\frac{1}{\rho_{i}\left(y_{i}(t)\right)} y_{i}^{\prime}(t)\right) \operatorname{sign}\left(x_{i}(t)-y_{i}(t)\right) \\
& =\left(-b_{i}\left(t, x_{i}(t)\right)-\sum_{j=1}^{n} f_{i j}\left(t, x_{j, t}\right)+b_{i}\left(t, y_{i}(t)\right)+\sum_{j=1}^{n} f_{i j}\left(t, y_{j, t}\right)\right) \operatorname{sign}\left(x_{i}(t)-y_{i}(t)\right) \\
& =h_{i}\left(t, x_{t}, y_{t}\right) \operatorname{sign}\left(x_{i}(t)-y_{i}(t)\right)
\end{aligned}
$$


where $h_{i}(t, \varphi, \psi):=-b_{i}\left(t, \varphi_{i}(0)\right)-\sum_{j=1}^{n} f_{i j}\left(t, \varphi_{j}\right)+b_{i}\left(t, \psi_{i}(0)\right)+\sum_{j=1}^{n} f_{i j}\left(t, \psi_{j}\right)$ for $t \geq 0$ and $\varphi, \psi \in U C_{g}$.

Lemma 3.2. Assume (A1)-(A4).

Then, for each $t_{0} \geq 0$ and $\varphi, \psi \in B C$, we have

$$
|V(t)|=\left|V\left(t, t_{0}, \varphi, \psi\right)\right| \leq \max _{j}\left\{\frac{1}{\underline{\rho}_{j}}\right\}\|\varphi-\psi\|, \quad \forall t \geq t_{0} .
$$

for $V(t)$ defined by (3.4).

Proof. Let $t_{0} \geq 0, \varphi, \psi \in B C, x(t)$ and $y(t)$ be the solutions of (3.1) such that $x_{t_{0}}=\varphi$ and $y_{t_{0}}=\psi$ respectively, and $V(t)$ defined by (3.4).

Suppose that the inequality (3.6) does not hold. As $V(t)$ is a positive continuous vector function and, for each $i \in\{1, \ldots, n\}, V_{i}\left(t_{0}\right) \leq \frac{1}{\underline{\rho}_{i}}\left|x_{i}\left(t_{0}\right)-y_{i}\left(t_{0}\right)\right| \leq \max _{j}\left\{\frac{1}{\underline{\rho}_{j}}\right\}\|\varphi-\psi\|$, then there is $t_{1}>t_{0}$ such that $\left|V\left(t_{1}\right)\right|>\max _{j}\left\{\frac{1}{\underline{\rho}_{j}}\right\}\|\varphi-\psi\|$. Defining

$$
T:=\min \left\{t \in\left[t_{0}, t_{1}\right]:|V(t)|=\max _{s \in\left[t_{0}, t_{1}\right]}|V(s)|\right\}
$$

and choosing $i \in\{1, \ldots, n\}$ such that $V_{i}(T)=|V(T)|$, we have $V_{i}(T)>0, V_{i}^{\prime}(T) \geq 0$ and $V_{i}(T)>$ $|V(t)|$ for all $t<T$.

On the other hand, by hypotheses (A1)-(A4) and (3.5), we have

$$
\begin{aligned}
V_{i}^{\prime}(T) & =h_{i}\left(T, x_{T}, y_{T}\right) \operatorname{sign}\left(x_{i}(T)-y_{i}(T)\right) \\
& \leq\left(b_{i}\left(T, y_{i}(T)\right)-b_{i}\left(T, x_{i}(T)\right)\right) \operatorname{sign}\left(x_{i}(T)-y_{i}(T)\right)+\sum_{j=1}^{n}\left|f_{i j}\left(T, x_{j, T}\right)-f_{i j}\left(T, y_{j, T}\right)\right| \\
& \leq-\beta_{i}(T)\left|x_{i}(T)-y_{i}(T)\right|+\sum_{j=1}^{n} l_{i j}(T)\left\|x_{j, T}-y_{j, T}\right\| \\
& \leq-\underline{\rho}_{i} \beta_{i}(T) V_{i}(T)+\sum_{j=1}^{n} l_{i j}(T) \max \left\{\|\varphi-\psi\|, \bar{\rho}_{j} \sup _{t_{0}-T<s \leq 0} V_{j}(T+s)\right\} \\
& =-\underline{\rho}_{i} \beta_{i}(T) V_{i}(T)+\sum_{j=1}^{n} l_{i j}(T) \bar{\rho}_{j} \max \left\{\frac{\|\varphi-\psi\|}{\bar{\rho}_{j}}, \sup _{t_{0}-T<s \leq 0} V_{j}(T+s)\right\}
\end{aligned}
$$

and, since $V_{i}(T) \geq \max _{j}\left\{\frac{\|\varphi-\psi\|}{\underline{\rho}_{j}}, V_{j}(t)\right\}$ for all $t \in\left[t_{0}, T\right]$, we conclude that

$$
V_{i}^{\prime}(T) \leq\left(-\underline{\rho}_{i} \beta_{i}(T)+\sum_{j=1}^{n} \bar{\rho}_{j} l_{i j}(T)\right) V_{i}(T)<0,
$$

which is a contradiction.

Proposition 3.3. Assume (A1)-(A4).

Then the system (3.1) is stable. 
Proof. Let $t_{0}>0$ and $\varepsilon>0$. Choosing $\delta=\varepsilon\left(\max _{i}\left\{\bar{\rho}_{i}\right\} \max _{j}\left\{\frac{1}{\underline{\rho}_{j}}\right\}\right)^{-1}>0$, from Lemma 3.2 we have, for any $\varphi, \psi \in B C$ such that $\|\varphi-\psi\|<\delta$,

$$
\begin{aligned}
\left|x\left(t, t_{0}, \varphi\right)-x\left(t, t_{0}, \psi\right)\right| & =\max _{i}\left\{\bar{\rho}_{i} \frac{1}{\bar{\rho}_{i}}\left|x_{i}\left(t, t_{0}, \varphi\right)-x_{i}\left(t, t_{0}, \psi\right)\right|\right\} \\
& \leq \max _{i}\left\{\bar{\rho}_{i}\left|\int_{x_{i}\left(t, t_{0}, \psi\right)}^{x_{i}\left(t, t_{0}, \varphi\right)} \frac{1}{\rho_{i}(s)} d s\right|\right\}=\max _{i}\left\{\bar{\rho}_{i} V_{i}\left(t, t_{0}, \varphi, \psi\right)\right\} \\
& \leq \max _{i}\left\{\bar{\rho}_{i}\right\}\left|V\left(t, t_{0}, \varphi, \psi\right)\right| \leq \max _{i}\left\{\bar{\rho}_{i}\right\} \max _{j}\left\{\frac{1}{\underline{\rho}_{j}}\right\}\|\varphi-\psi\| \\
& <\varepsilon
\end{aligned}
$$

for all $t \geq t_{0}$.

Remark 3.1. We note that the Lemmas 3.1 and 3.2, and Proposition 3.3 are also true if we assume

$$
\left|f_{i j}(t, \varphi)-f_{i j}(t, \psi)\right| \leq l_{i j}(t)\|\varphi-\psi\|, \quad \forall t \geq 0, \forall \varphi, \psi \in B C^{1}
$$

instead of (A3).

Now, we state our main result on the global asymptotic stability of system (3.1).

Theorem 3.4. Assume (A1)-(A4). If

$$
\limsup _{t \rightarrow+\infty} \sum_{j=1}^{n} \frac{\bar{\rho}_{j}}{\underline{\rho}_{i}} \frac{l_{i j}(t)}{\beta_{i}(t)}<1,
$$

then the system (3.1) is globally asymptotically stable.

Proof. From Proposition 3.3, we know that (3.1) is stable. It remains to prove the asymptotic stability.

Let $t_{0}>0$ and $x(t)=x\left(t, t_{0}, \varphi\right), y(t)=x\left(t, t_{0}, \psi\right)$ be solutions of (3.1) with $\varphi, \psi \in B C$. Defining $V:\left[t_{0},+\infty\right) \rightarrow \mathbb{R}^{n}$ by $(3.4)$, we know that

$$
\lim _{t \rightarrow+\infty}(x(t)-y(t))=0 \Leftrightarrow \lim _{t \rightarrow+\infty} V(t)=0 .
$$

From Lemma 3.2, we know that $t \mapsto V(t)$ is a bounded function and it is possible to define the limits

$$
u_{i}:=\limsup _{t \rightarrow+\infty} V_{i}(t), \quad i=1, \ldots, n,
$$

and

$$
u:=\max _{i}\left\{u_{i}\right\} .
$$

Since $V(t) \geq 0$ for all $t \geq t_{0}$, then we have $u \in[0,+\infty)$. From (3.8), it remains to prove that $u=0$.

Let $i \in\{1, \ldots, n\}$ be such that $u_{i}=u$. Now we prove that there is a sequence $\left(t_{k}\right)_{k \in \mathbb{N}}$ such that

$$
t_{k} \nearrow+\infty, \quad V_{i}\left(t_{k}\right) \rightarrow u, \quad \text { and } \quad V_{i}^{\prime}\left(t_{k}\right) \rightarrow 0, \quad \text { as } \quad k \rightarrow+\infty .
$$

Case 1. Assume that $V_{i}(t)$ is eventually monotone. In this case, $\lim _{t \rightarrow+\infty} V_{i}(t)=u$ and, for large $t$, $V_{i}(t)$ is a differentiable, monotone and bounded real function. Hence there is a sequence $\left(t_{k}\right)_{k \in \mathbb{N}}$ such that $t_{k} \nearrow+\infty$ and $V_{i}^{\prime}\left(t_{k}\right) \rightarrow 0$. 
Case 2. Assume that $V_{i}(t)$ is not eventually monotone. In this case there is a sequence $\left(t_{k}\right)_{k \in \mathbb{N}}$ such that $t_{k} \nearrow+\infty, V_{i}^{\prime}\left(t_{k}\right)=0$, and $V_{i}\left(t_{k}\right) \rightarrow u$, as $k \rightarrow+\infty$ and (3.9) holds.

For the sake of contradiction, assume that $u>0$.

Fix $\varepsilon>0$ and let $T=T(\varepsilon)>t_{0}$ be such that $\varepsilon<u, \frac{\|\varphi-\psi\|}{\bar{\rho}_{j} g\left(t_{0}-T\right)}<u+\varepsilon$ for all $j \in\{1, \ldots, n\}$, and $|V(t)|<u+\varepsilon$ for all $t \geq T$. Defining $\mathcal{V}:=\max _{s \in\left[t_{0}, T\right]}|V(s)|$, from (g3) we conclude that there is $s_{0}<0$ such that $\frac{\mathcal{V}}{g(s)}<u+\varepsilon$ for all $s<s_{0}$. Thus, for $t>T-s_{0}$, we have

$$
\begin{aligned}
\sup _{s \in\left[t_{0}-t, 0\right]} \frac{|V(t+s)|}{g(s)} & \leq \max \left\{\sup _{s \in\left[t_{0}-t, T-t\right]} \frac{|V(t+s)|}{g(s)}, \sup _{s \in[T-t, 0]} \frac{|V(t+s)|}{g(s)}\right\} \\
& \leq \max \left\{\sup _{s \in\left[t_{0}-t, T-t\right]} \frac{\mathcal{V}}{g(s)}, \sup _{s \in[T-t, 0]} \frac{u+\varepsilon}{g(s)}\right\} \leq u+\varepsilon .
\end{aligned}
$$

Now, from the hypotheses (A1)-(A3), the definition (3.4), and (3.5), we have, for $t_{k}>T-s_{0}$,

$$
\begin{aligned}
V_{i}^{\prime}\left(t_{k}\right) & =h_{i}\left(t_{k}, x_{t_{k}}, y_{t_{k}}\right) \operatorname{sign}\left(x_{i}\left(t_{k}\right)-y_{i}\left(t_{k}\right)\right) \\
& \leq\left(b_{i}\left(t_{k}, y_{i}\left(t_{k}\right)\right)-b_{i}\left(t_{k}, x_{i}\left(t_{k}\right)\right)\right) \operatorname{sign}\left(x_{i}\left(t_{k}\right)-y_{i}\left(t_{k}\right)\right)+\sum_{j=1}^{n}\left|f_{i j}\left(t_{k}, x_{j, t_{k}}\right)-f_{i j}\left(t_{k}, y_{j, t_{k}}\right)\right| \\
& \leq-\beta_{i}\left(t_{k}\right)\left|x_{i}\left(t_{k}\right)-y_{i}\left(t_{k}\right)\right|+\sum_{j=1}^{n} l_{i j}\left(t_{k}\right)\left\|x_{j, t_{k}}-y_{j, t_{k}}\right\|_{g} \\
& \leq-\underline{\rho}_{i} \beta_{i}\left(t_{k}\right) V_{i}\left(t_{k}\right)+\sum_{j=1}^{n} \bar{\rho}_{j} l_{i j}\left(t_{k}\right) \max \left\{\frac{\|\varphi-\psi\|}{\bar{\rho}_{j} g\left(t_{0}-t_{k}\right)}, \sup _{s \in\left[t_{0}-t_{k}, 0\right]} \frac{V_{j}\left(t_{k}+s\right)}{g(s)}\right\} \\
& \leq-\underline{\rho}_{i} \beta_{i}\left(t_{k}\right) V_{i}\left(t_{k}\right)+\sum_{j=1}^{n} \bar{\rho}_{j} l_{i j}\left(t_{k}\right)(u+\varepsilon),
\end{aligned}
$$

which implies that

$$
V_{i}\left(t_{k}\right) \leq-\frac{V_{i}^{\prime}\left(t_{k}\right)}{\underline{\rho}_{i} \beta_{i}\left(t_{k}\right)}+\sum_{j=1}^{n} \frac{\bar{\rho}_{j}}{\underline{\rho}_{i}} \frac{l_{i j}\left(t_{k}\right)}{\beta_{i}\left(t_{k}\right)}(u+\varepsilon) .
$$

From (3.9) we have $V_{i}\left(t_{k}\right) \rightarrow u$ and $V_{i}^{\prime}\left(t_{k}\right) \rightarrow 0$ as $k \rightarrow+\infty$ and, letting $\varepsilon \rightarrow 0$ and $k \rightarrow+\infty$, from (3.10) and (A4), we get

$$
u \leq\left(\limsup _{t \rightarrow+\infty} \sum_{j=1}^{n} \frac{\bar{\rho}_{j}}{\underline{\rho}_{i}} \frac{l_{i j}(t)}{\beta_{i}(t)}\right) u<u,
$$

which is a contradiction.

The next result shows that the same conclusion can be obtained if we assume (A4') there exist $\alpha>0$ and $d=\left(d_{1}, \ldots, d_{n}\right)>0$ such that, for each $i \in\{1, \ldots, n\}$,

$$
\underline{\rho}_{i} d_{i} \beta_{i}(t)-\sum_{j=1}^{n} \bar{\rho}_{j} d_{j} l_{i j}(t)>\alpha, \forall t \geq 0 \quad \text { and } \quad \limsup _{t \rightarrow+\infty} \sum_{j=1}^{n} \frac{\bar{\rho}_{j}}{\rho_{i}} \frac{d_{j} l_{i j}(t)}{d_{i} \beta_{i}(t)}<1,
$$


instead of (A4) and (3.7) respectively.

Corollary 3.5. Assume (A1)-(A3) and (A4'). Then the system (3.1) is globally asymptotically stable.

Proof. The change of variables $y_{i}(t)=d_{i}^{-1} x_{i}(t)$ transforms (3.1) into

$$
y_{i}^{\prime}(t)=-\rho_{i}\left(d_{i} y_{i}(t)\right) d_{i}^{-1}\left[b_{i}\left(t, d_{i} y_{i}(t)\right)+\sum_{j=1}^{n} f_{i j}\left(t, d_{j} y_{j, t}\right)\right], t \geq 0, i=1, \ldots, n .
$$

Defining, for each $i, j \in\{1, \ldots, n\}, \tilde{\rho}_{i}(u):=\rho_{i}\left(d_{i} u\right), \tilde{b}_{i}(t, u):=d_{i}^{-1} b_{i}\left(t, d_{i} u\right)$, and $\tilde{f}_{i j}(t, \varphi):=$ $d_{i}^{-1} f_{i j}\left(t, d_{j} \varphi\right)$, for all $t \geq 0, u \in \mathbb{R}, \varphi \in B C^{1}$, the system (3.12) has the form

$$
y_{i}^{\prime}(t)=-\tilde{\rho}_{i}\left(y_{i}(t)\right)\left[\tilde{b}_{i}\left(t, y_{i}(t)\right)+\sum_{i=1}^{n} \tilde{f}_{i j}\left(t, y_{j, t}\right)\right], t \geq 0, i=1, \ldots, n
$$

where $\tilde{\rho}_{i}$ satisfies (A1), with $\underline{\tilde{\rho}}_{i}=\underline{\rho}_{i}$ and $\overline{\tilde{\rho}}_{i}=\bar{\rho}_{i}$, and $\tilde{b}_{i}$ satisfies (A2) with $\tilde{\beta}_{i}(t)=\beta_{i}(t)$. Moreover, from (A3), for $\varphi, \psi \in B C^{1}$ we have

$$
\left|\tilde{f}_{i j}(t, \varphi)-\tilde{f}_{i j}(t, \psi)\right|=d_{i}^{-1}\left|f_{i j}\left(t, d_{j} \varphi\right)-f_{i j}\left(t, d_{j} \psi\right)\right| \leq \frac{d_{j}}{d_{i}} l_{i j}(t)\|\varphi-\psi\|_{g}
$$

which implies that $\tilde{f}_{i j}$ satisfies (A3) with the function $\tilde{l}_{i j}(t):=d_{i}^{-1} d_{j} l_{i j}(t)$, for all $i, j \in\{1, \ldots, n\}$. Thus, from (A4') conditions (A4) and (3.7) hold and the conclusion follows from Theorem 3.4.

Finally, it is easy to see that, if $l_{i j}(t)$ are bounded functions then the hypothesis (A4) implies the condition (3.7) and the following result holds:

Corollary 3.6. Assume (A1)-(A4) where $l_{i j}(t)$ are bounded functions. Then the system (3.1) is globally asymptotically stable.

Now, in the phase space $U C_{g}$, we consider the following nonautonomous Cohen-Grossberg neural network model with both time-varying delays and distributed delays

$x_{i}^{\prime}(t)=-\rho_{i}\left(x_{i}(t)\right)\left[b_{i}\left(t, x_{i}(t)\right)+\sum_{j=1}^{n} h_{i j}\left(t, x_{j}\left(t-\tau_{i j 1}(t)\right), \ldots, x_{j}\left(t-\tau_{i j P}(t)\right)\right)+\sum_{j=1}^{n} f_{i j}\left(t, x_{j, t}\right)\right], t \geq 0$

$i=1, \ldots, n, P \in \mathbb{N}$, where the functions $\rho_{i}, b_{i}$, and $f_{i j}$ are as in the model $(3.1)$ and $h_{i j}:[0,+\infty) \times$ $\mathbb{R}^{P} \rightarrow \mathbb{R}, \tau_{i j p}:[0,+\infty) \rightarrow[0,+\infty)$ are continuous functions such that, for each $i, j \in\{1, \ldots, n\}$, $p \in\{1, \ldots, P\}$,

$$
\begin{aligned}
& t-\tau_{i j p}(t) \rightarrow+\infty \text { as } t \rightarrow+\infty \\
& \left|h_{i j}(t, u)-h_{i j}(t, v)\right| \leq H_{i j}(t)|u-v|, \quad \forall t \geq 0, \forall u, v \in \mathbb{R}^{P},
\end{aligned}
$$

for some function $H_{i j}:[0,+\infty) \rightarrow[0,+\infty)$.

Using the same techniques presented in the proof of Theorem 3.4 we obtain the following stability result.

Theorem 3.7. Assume (A1), (A2), (A3), (3.15), and (3.16).

If there exist $\alpha>0$ and $d=\left(d_{1}, \ldots, d_{n}\right)>0$ such that, for each $i \in\{1, \ldots, n\}$,

$$
\begin{aligned}
& \underline{\rho}_{i} d_{i} \beta_{i}(t)-\sum_{j=1}^{n} \bar{\rho}_{j} d_{j}\left(H_{i j}(t)+l_{i j}(t)\right)>\alpha, \quad \forall t \geq 0, \\
& \limsup _{t \rightarrow+\infty} \sum_{j=1}^{n} \frac{\bar{\rho}_{j}}{\rho_{i}} \frac{d_{j}\left(H_{i j}(t)+l_{i j}(t)\right)}{d_{i} \beta_{i}(t)}<1,
\end{aligned}
$$

then the system (3.14) is globally asymptotically stable. 
Proof. As we saw in the proof of Corollary 3.5, we do not lose generality if we assume (3.17) and (3.18) with $d_{j}=1$ for all $j \in\{1, \ldots, n\}$.

Let $t_{0}>0$ and $x(t)=x\left(t, t_{0}, \varphi\right), y(t)=x\left(t, t_{0}, \psi\right)$ be solutions of (3.14) with $\varphi, \psi \in B C$. From Remark 3.1, we conclude that $x(t)$ and $y(t)$ are defined on $\mathbb{R}$ and the function $t \mapsto V(t)$, defined by (3.4), is bounded. Following the same ideas in the proof of Theorem 3.4, we conclude that, for $u=\max _{j}\left(\limsup _{t \rightarrow+\infty} V_{j}(t)\right)$, there is a sequence $\left(t_{k}\right)_{k}$ satisfying (3.9) and, for $\varepsilon>0, T=T(\varepsilon)>0$ in the same conditions, we have, for $t_{k}>T-s_{0}$ (see the notation in the proof of Theorem 3.4),

$$
\begin{aligned}
V_{i}^{\prime}\left(t_{k}\right) & \leq\left(b_{i}\left(t_{k}, y_{i}\left(t_{k}\right)\right)-b_{i}\left(t_{k}, x_{i}\left(t_{k}\right)\right)\right) \operatorname{sign}\left(x_{i}\left(t_{k}\right)-y_{i}\left(t_{k}\right)\right)+\sum_{j=1}^{n}\left|f_{i j}\left(t_{k}, x_{j, t_{k}}\right)-f_{i j}\left(t_{k}, y_{j, t_{k}}\right)\right| \\
& +\sum_{j=1}^{n}\left|h_{i j}\left(t_{k}, x_{j}\left(t_{k}-\tau_{i j 1}\left(t_{k}\right)\right), \ldots, x_{j}\left(t_{k}-\tau_{i j P}\left(t_{k}\right)\right)\right)-h_{i j}\left(t_{k}, y_{j}\left(t_{k}-\tau_{i j 1}\left(t_{k}\right)\right), \ldots, y_{j}\left(t_{k}-\tau_{i j P}\left(t_{k}\right)\right)\right)\right| \\
& \leq-\underline{\rho}_{i} \beta_{i}\left(t_{k}\right) V_{i}\left(t_{k}\right)+\sum_{j=1}^{n} \bar{\rho}_{j} l_{i j}\left(t_{k}\right)(u+\varepsilon)+\sum_{j=1}^{n} H_{i j}\left(t_{k}\right) \max _{p}\left|x_{j}\left(t_{k}-\tau_{i j p}\left(t_{k}\right)\right)-y_{j}\left(t_{k}-\tau_{i j p}\left(t_{k}\right)\right)\right| .
\end{aligned}
$$

As $\lim _{t \rightarrow+\infty}\left(t-\tau_{i j p}(t)\right)=+\infty$ and, for the sake of contradiction, we assume that $u>0$, then there is $S>t_{0}$ such that, for $t_{k}>S$, we have $t_{k}-\tau_{i j p}\left(t_{k}\right)>t_{0}$ and $V_{j}\left(t_{k}-\tau_{i j p}\left(t_{k}\right)\right)<u+\varepsilon$ for all $i, j \in\{1, \ldots, n\}, p \in\{1, \ldots, P\}$. Thus, for $t_{k}>\max \left\{T-s_{0}, S\right\}$, we have

$$
\begin{aligned}
V_{i}^{\prime}\left(t_{k}\right) & \leq-\underline{\rho}_{i} \beta_{i}\left(t_{k}\right) V_{i}\left(t_{k}\right)+\sum_{j=1}^{n} \bar{\rho}_{j} l_{i j}\left(t_{k}\right)(u+\varepsilon)+\sum_{j=1}^{n} H_{i j}\left(t_{k}\right) \bar{\rho}_{j} \max _{p} V_{j}\left(t_{k}-\tau_{i j p}\left(t_{k}\right)\right) \\
& \leq-\underline{\rho}_{i} \beta_{i}\left(t_{k}\right) V_{i}\left(t_{k}\right)+\sum_{j=1}^{n} \bar{\rho}_{j}\left(H_{i j}\left(t_{k}\right)+l_{i j}\left(t_{k}\right)\right)(u+\varepsilon),
\end{aligned}
$$

which implies that

$$
V_{i}\left(t_{k}\right) \leq-\frac{V_{i}^{\prime}\left(t_{k}\right)}{\underline{\rho}_{i} \beta_{i}\left(t_{k}\right)}+\sum_{j=1}^{n} \frac{\bar{\rho}_{j}}{\underline{\rho}_{i}} \frac{H_{i j}\left(t_{k}\right)+l_{i j}\left(t_{k}\right)}{\beta_{i}\left(t_{k}\right)}(u+\varepsilon) .
$$

Since $V_{i}\left(t_{k}\right) \rightarrow u$ and $V_{i}^{\prime}\left(t_{k}\right) \rightarrow 0$ as $k \rightarrow+\infty$, then letting $\varepsilon \rightarrow 0$ and $k \rightarrow+\infty$, from (3.19) and (3.18), we get

$$
u \leq\left(\limsup _{t \rightarrow+\infty} \sum_{j=1}^{n} \frac{\bar{\rho}_{j}}{\underline{\rho}_{i}} \frac{H_{i j}(t)+l_{i j}(t)}{\beta_{i}(t)}\right) u<u,
$$

which is a contradiction.

\section{Applications}

Here, we apply the previous results to the following nonautonomous Cohen-Grossberg neural network model with both time-varying delays and distributed delays:

$$
\begin{aligned}
x_{i}^{\prime}(t)=-\rho_{i}\left(x_{i}(t)\right)[ & b_{i}\left(t, x_{i}(t)\right)+\sum_{j=1}^{n} \sum_{p=1}^{P}\left(a_{i j p}(t) h_{i j p}\left(x_{j}\left(t-\tau_{i j p}(t)\right)\right)\right. \\
& \left.\left.\quad+c_{i j p}(t) f_{i j p}\left(\int_{-\infty}^{0} g_{i j p}\left(x_{j}(t+s)\right) d \eta_{i j p}(s)\right)\right)+I_{i}(t)\right], t \geq 0, i=1, \ldots, n,
\end{aligned}
$$


where $\rho_{i}: \mathbb{R} \rightarrow(0,+\infty), b_{i}:[0,+\infty) \times \mathbb{R} \rightarrow \mathbb{R}, a_{i j p}, c_{i j p}, I_{i}:[0,+\infty) \rightarrow \mathbb{R}, h_{i j p}, f_{i j p}, g_{i j p}: \mathbb{R} \rightarrow \mathbb{R}$, and $\tau_{i j p}:[0,+\infty) \rightarrow[0,+\infty)$ are continuous functions, with $t-\tau_{i j p}(t) \rightarrow+\infty$ as $t \rightarrow+\infty$ and $\eta_{i j p}:(-\infty, 0] \rightarrow \mathbb{R}$ are non-decreasing, bounded, and normalized, i.e. $\eta_{i j p}(0)-\eta_{i j p}(-\infty)=1$, for all $i, j \in\{1, \ldots, n\}, p \in\{1, \ldots, P\}$. For (4.1), in the sequel we further assume that the functions $\rho_{i}$ satisfy (A1), $b_{i}$ satisfy (A2), and $h_{i j p}, f_{i j p}, g_{i j p}$ are Lipschitzian with Lipschitz constants $\xi_{i j p}, \mu_{i j p}$, $\sigma_{i j p}$ respectively.

Model (4.1) is a generalization of a model introduced in [20] and it is particularly relevant in terms of applications, since it includes different types of neural network models as subclasses, as we shall illustrate with several examples.

Before to give a global stability criterion for system (4.1), we need an auxiliary lemma, established in [8], to define a function $g$ satisfying (g1)-(g3) required to build the convenient phase space of (4.1). For convenience of the reader, we put the proof here.

Lemma 4.1. [8] Consider $\eta_{i}:(-\infty, 0] \rightarrow \mathbb{R}, i=1, \ldots, m$ non-decreasing and bounded functions, and $\alpha>0$ such that

$$
\int_{-\infty}^{0} d \eta_{i}(s)<\alpha, \quad i=1, \ldots, m .
$$

Then, there is a continuous function $g:(-\infty, 0] \rightarrow[1,+\infty)$ satisfying (g1)-(g3), and such that

$$
\int_{-\infty}^{0} g(s) d \eta_{i}(s)<\alpha, \quad i=1, \ldots, m .
$$

Proof. First, define

$$
\alpha_{i}:=\eta_{i}(0)-\eta_{i}(-\infty)=\int_{-\infty}^{0} d \eta_{i}(s)<\alpha, \quad i=1, \ldots, m .
$$

For each $n \in \mathbb{N}$ and $i \in\{1, \ldots, m\}$, let $\varepsilon_{i, n}=\left(\alpha-\alpha_{i}\right) /\left[2^{n+1}(n+1)\right]$. Since $\eta_{i}$ is non-decreasing and bounded, there is a sequence $\left(r_{n}\right)_{n \in \mathbb{N}}$ of positive real numbers (independent of $i$ ) such that $r_{n+1} \geq r_{n}+1$ and

$$
\int_{-\infty}^{-r_{n}} d \eta_{i}(s)<\varepsilon_{i, n}, \quad i=1, \ldots, m, n \in \mathbb{N} .
$$

Now, define $g:(-\infty, 0] \rightarrow[1,+\infty)$ as follows:

(i) $g(s)=1$ on $\left[-r_{1}, 0\right]$;

(ii) $g\left(-r_{n}\right)=n, n \in \mathbb{N}$;

(iii) $g$ is continuous and piecewise linear (linear on intervals $\left[-r_{n+1},-r_{n}\right]$ ).

From (i) and (4.2), we have

$$
\int_{-r_{1}}^{0} g(s) d \eta_{i}(s)<\frac{\alpha+\alpha_{i}}{2} .
$$

Hence, for each $i=1, \ldots, m$, we have

$$
\begin{aligned}
\int_{-\infty}^{0} g(s) d \eta_{i}(s) & =\int_{-r_{1}}^{0} g(s) d \eta_{i}(s)+\sum_{n=1}^{\infty} \int_{-r_{n+1}}^{-r_{n}} g(s) d \eta_{i}(s)<\frac{\alpha+\alpha_{i}}{2}+\sum_{n=1}^{\infty} g\left(-r_{n+1}\right) \int_{-r_{n+1}}^{-r_{n}} d \eta_{i}(s) \\
& \leq \frac{\alpha+\alpha_{i}}{2}+\sum_{n=1}^{\infty}(n+1) \varepsilon_{i, n}=\frac{\alpha+\alpha_{i}}{2}+\sum_{n=1}^{\infty} \frac{\alpha-\alpha_{i}}{2^{n+1}}=\alpha .
\end{aligned}
$$


Theorem 4.2. Consider (4.1) under the hypotheses assumed above.

If (3.17) and (3.18) hold with $H_{i j}(t):=\sum_{p=1}^{P}\left|a_{i j p}(t)\right| \xi_{i j p}$ and $l_{i j}(t):=\sum_{p=1}^{P}\left|c_{i j p}(t)\right| \mu_{i j p} \sigma_{i j p}$, then the system (4.1) is globally asymptotically stable.

Proof. As in the proof of Corollary 3.5, after a change of variable, we may assume that $d_{i}=1$ for all $i \in\{1, \ldots, n\}$.

On the one hand, from (3.18), with $H_{i j}(t)=\sum_{p=1}^{P}\left|a_{i j p}(t)\right| \xi_{i j p}$ and $l_{i j}(t)=\sum_{p=1}^{P}\left|c_{i j p}(t)\right| \mu_{i j p} \sigma_{i j p}$, it is easy to verify that there exists $\delta_{1}>0$ such that

$$
\limsup _{t \rightarrow+\infty} \sum_{j=1}^{n} \frac{\sum_{\rho_{j}}}{\underline{\rho}_{i}} \frac{\sum_{p=1}^{P}\left|a_{i j p}(t)\right| \xi_{i j p}+\sum_{p=1}^{P}\left|c_{i j p}(t)\right| \mu_{i j p} \sigma_{i j p}\left(1+\delta_{1}\right)}{\beta_{i}(t)}<1 .
$$

On the other hand, the condition (3.17), with $H_{i j}(t)=\sum_{p=1}^{P}\left|a_{i j p}(t)\right| \xi_{i j p}$ and $l_{i j}(t)=\sum_{p=1}^{P}\left|c_{i j p}(t)\right| \mu_{i j p} \sigma_{i j p}$, is equivalent to

$$
\underline{\rho}_{i} \beta_{i}(t)-\sum_{j=1}^{n} \bar{\rho}_{j}\left(H_{i j}(t)+l_{i j}(t)\right)>0 \forall t \geq 0 \text { and } \liminf _{t \rightarrow+\infty}\left(\underline{\rho}_{i} \beta_{i}(t)-\sum_{j=1}^{n} \bar{\rho}_{j}\left(H_{i j}(t)+l_{i j}(t)\right)\right)>0
$$

from which, together with (3.18), we obtain that there exists $\delta_{2}>0$ such that

$\underline{\rho}_{i} \beta_{i}(t)-\sum_{j=1}^{n} \bar{\rho}_{j}\left(H_{i j}(t)+l_{i j}(t)\left(1+\delta_{2}\right)\right)>0 \forall t \geq 0$ and $\liminf _{t \rightarrow+\infty}\left(\underline{\rho}_{i} \beta_{i}(t)-\sum_{j=1}^{n} \bar{\rho}_{j}\left(H_{i j}(t)+l_{i j}(t)\left(1+\delta_{2}\right)\right)\right)>0 .(4.4)$

Considering $\delta:=\min \left\{\delta_{1}, \delta_{2}\right\}$, from (4.3) and (4.4) we conclude that there is $\gamma>0$ such that, for each $i \in\{1, \ldots, n\}$,

$$
\underline{\rho}_{i} \beta_{i}(t)-\sum_{j=1}^{n} \bar{\rho}_{j}\left(\sum_{p=1}^{P}\left|a_{i j p}(t)\right| \xi_{i j p}+\sum_{p=1}^{P}\left|c_{i j p}(t)\right| \mu_{i j p} \sigma_{i j p}(1+\delta)\right)>\gamma, \quad \forall t \geq 0,
$$

and

$$
\limsup _{t \rightarrow+\infty} \sum_{j=1}^{n} \frac{\sum_{\rho_{j}}}{\underline{\rho}_{i}} \frac{\sum_{p=1}^{P}\left|a_{i j p}(t)\right| \xi_{i j p}+\sum_{p=1}^{P}\left|c_{i j p}(t)\right| \mu_{i j p} \sigma_{i j p}(1+\delta)}{\beta_{i}(t)}<1
$$

Since $\int_{-\infty}^{0} d \eta_{i j p}(s)<1+\delta$ for $i, j \in\{1, \ldots, n\}, p \in\{1, \ldots, P\}$, from Lemma 4.1 , there is $g:(-\infty, 0] \stackrel{\infty}{\rightarrow}[1, \infty)$ satisfying (g1)-(g3) such that

$$
\int_{-\infty}^{0} g(s) d \eta_{i j p}(s)<1+\delta, \quad \forall i, j \in\{1, \ldots, n\}, p \in\{1, \ldots, P\}
$$

and we consider $U C_{g}$ as the phase space of (4.1). Defining, for each $i, j \in\{1, \ldots, n\}$,

$$
h_{i j}\left(t, u_{1}, \ldots, u_{P}\right):=\sum_{p=1}^{P} a_{i j p}(t) h_{i j p}\left(u_{p}\right)
$$


and

$$
f_{i j}\left(t, \phi_{j}\right):=\sum_{p=1}^{P} c_{i j p}(t) f_{i j p}\left(\int_{-\infty}^{0} g_{i j p}\left(\phi_{j}(s)\right) d \eta_{i j p}(s)\right)+I_{i}(t),
$$

with $\left(u_{1}, \ldots, u_{P}\right) \in \mathbb{R}^{P}, \phi=\left(\phi_{1}, \ldots, \phi_{n}\right) \in U C_{g}$, and $t \geq 0$, the system (4.1) has the form (3.14).

As $h_{i j p}$ are Lipschitz functions with Lipschitz constant $\xi_{i j p}$, then $(3.16)$ holds with $H_{i j}(t)=$ $\sum_{p=1}^{P}\left|a_{i j p}(t)\right| \xi_{i j p}$.

For $\phi, \varphi \in B C^{1}$ and $t \geq 0$, since $f_{i j p}, g_{i j p}$ are Lipschitz functions and $\eta_{i j p}$ are non-decreasing, we have, for each $i, j \in\{1, \ldots, n\}$,

$$
\begin{aligned}
\left|f_{i j}(t, \phi)-f_{i j}(t, \varphi)\right| & \leq \sum_{p=1}^{P}\left|c_{i j p}(t)\right| \mu_{i j p} \int_{-\infty}^{0}\left|g_{i j p}(\phi(s))-g_{i j p}(\varphi(s))\right| d \eta_{i j p}(s) \\
& \leq \sum_{p=1}^{P}\left|c_{i j p}(t)\right| \mu_{i j p} \sigma_{i j p} \int_{-\infty}^{0} g(s) \frac{|(\phi-\varphi)(s)|}{g(s)} d \eta_{i j p}(s) \\
& \leq\left(\sum_{p=1}^{P}\left|c_{i j p}(t)\right| \mu_{i j p} \sigma_{i j p} \int_{-\infty}^{0} g(s) d \eta_{i j p}(s)\right)\|\phi-\varphi\|_{g} .
\end{aligned}
$$

This means that

$$
\left|f_{i j}(t, \phi)-f_{i j}(t, \varphi)\right| \leq\left(\sum_{p=1}^{P}\left|c_{i j p}(t)\right| \mu_{i j p} \sigma_{i j p}(1+\delta)\right)\|\phi-\varphi\|_{g},
$$

and, together with (4.5) and (4.6), the hypotheses (A3), (3.16), (3.17), and (3.18) hold. Now, the conclusion follows from Theorem 3.7.

To the best of our knowledge, the global asymptotic stability of the Cohen-Grossberg neural network model (4.1), without assuming the existence of an equilibrium point and with potentially unbounded coefficients, was never studied before and Theorem 4.2 establishes a new stability criterion.

Example 4.1. A particular example of (4.1) is the following Cohen-Grossberg neural network model

$$
\begin{aligned}
x_{i}^{\prime}(t)=-\rho_{i}\left(x_{i}(t)\right)[ & b_{i}\left(t, x_{i}(t)\right)-\sum_{j=1}^{n}\left(c_{i j}(t) \int_{-\tau_{i j}}^{0} f_{j}\left(x_{j}(t+s)\right) d \varsigma_{i j}(s)\right. \\
& \left.\left.\quad+d_{i j}(t) \int_{-\infty}^{0} g_{j}\left(x_{j}(t+s)\right) d \eta_{i j}(s)\right)+I_{i}(t)\right], t \geq 0, i=1, \ldots, n,
\end{aligned}
$$

which its global asymptotic stability was studied in [16] under a different set of hypotheses, including bounded coefficients $c_{i j}(t)$ and $d_{i j}(t)$. From Theorem 4.2 we have

Corollary 4.3. Assume that $\eta_{i j}:(-\infty, 0] \rightarrow \mathbb{R}, \varsigma_{i j}:\left[-\tau_{i j}, 0\right] \rightarrow \mathbb{R}$ are non-decreasing, bounded, and normalized functions, $f_{j}, g_{j}: \mathbb{R} \rightarrow \mathbb{R}$ are Lipschitz functions with Lipschitz constants $\mu_{j}, \sigma_{j}$ respectively, and $\rho_{i}: \mathbb{R} \rightarrow(0,+\infty), b_{i}:[0,+\infty) \times \mathbb{R} \rightarrow \mathbb{R}, c_{i j}, d_{i j}, I_{i}:[0,+\infty) \rightarrow \mathbb{R}$ are continuous functions such that $\rho_{i}$ and $b_{i}$ satisfy (A1) and (A2) respectively.

If there exist $\alpha>0$ and $d=\left(d_{1}, \ldots, d_{n}\right)>0$ such that, for each $i \in\{1, \ldots, n\}$,

$$
\begin{aligned}
& \underline{\rho}_{i} d_{i} \beta_{i}(t)-\sum_{j=1}^{n} \bar{\rho}_{j} d_{j}\left(\left|c_{i j}(t)\right| \mu_{j}+\left|d_{i j}(t)\right| \sigma_{j}\right)>\alpha, \quad \forall t \geq 0, \\
& \limsup _{t \rightarrow+\infty} \sum_{j=1}^{n} \frac{\bar{\rho}_{j}}{\underline{\rho}_{i}} \frac{d_{j}\left(\left|c_{i j}(t)\right| \mu_{j}+\left|d_{i j}(t)\right| \sigma_{j}\right)}{d_{i} \beta_{i}(t)}<1,
\end{aligned}
$$


then the system (4.7) is globally asymptotically stable.

Example 4.2. As we refereed above, the model (4.1) includes other types of neural network models. For example, if $\rho_{i}(t)=1, b_{i}(t, u)=c_{i}(t) u, P=2, \tau_{i j 1}(t)=0, a_{i j 1}(t)=a_{i j}(t), a_{i j 2}(t)=b_{i j}(t)$, $h_{i j 1}(u)=h_{i j 2}(u)=-f_{j}(u)$, and $c_{i j 1}(t)=c_{i j 2}(t)=0$ for all $t \geq 0$ and $u \in \mathbb{R}$, then system (4.1) reduces to the following Hopfield neural network model

$x_{i}^{\prime}(t)=-c_{i}(t) x_{i}(t)+\sum_{j=1}^{n} a_{i j}(t) f_{j}\left(x_{j}(t)\right)+\sum_{j=1}^{n} b_{i j}(t) f_{j}\left(x_{j}\left(t-\tau_{i j}(t)\right)\right)+I_{i}(t), t \geq 0, i=1, \ldots, n$.

Consequently, Theorem 4.2 applied to system (4.8) gives the following result.

Corollary 4.4. Assume that $a_{i j}, b_{i j}, c_{i}, I_{i}:[0,+\infty) \rightarrow \mathbb{R}, \tau_{i j}:[0,+\infty) \rightarrow[0,+\infty)$, and $f_{j}: \mathbb{R} \rightarrow \mathbb{R}$ are continuous functions such that

(i) $t-\tau_{i j}(t) \rightarrow+\infty$ as $t \rightarrow+\infty$;

(ii) $f_{j}: \mathbb{R} \rightarrow \mathbb{R}$ are $\mu_{j}$-Lipschitz functions;

(iii) there exist $d=\left(d_{1}, \ldots, d_{n}\right)>0$ and $\alpha>0$ such that, for each $i \in\{1, \ldots, n\}$,

$$
\begin{aligned}
& d_{i} c_{i}(t)-\sum_{j=1}^{n} d_{j} \mu_{j}\left(\left|a_{i j}(t)\right|+\left|b_{i j}(t)\right|\right)>\alpha, \quad \forall t \geq 0 ; \\
& \limsup _{t \rightarrow+\infty} \sum_{j=1}^{n} \frac{d_{j} \mu_{j}\left(\left|a_{i j}(t)\right|+\left|b_{i j}(t)\right|\right)}{d_{i} c_{i}(t)}<1 .
\end{aligned}
$$

Then the system (4.8) is globally asymptotically stable.

Remark 4.1. For system (4.8), Z. Yuan et al. [22] obtained the global asymptotic stability assuming the following hypotheses:

(a) $t-\tau_{i j}(t) \rightarrow+\infty$ as $t \rightarrow+\infty$;

(b) $f_{j}: \mathbb{R} \rightarrow \mathbb{R}$ are $\mu_{j}$-Lipschitz functions;

(c) $c_{i}(t), a_{i j}(t), b_{i j}(t)$, and $I_{i}(t)$ are bounded continuous functions;

(d) there exists $d=\left(d_{1}, \ldots, d_{n}\right)>0$ such that, for each $j \in\{1, \ldots, n\}$,

$$
\limsup _{t \rightarrow+\infty} \sum_{i=1}^{n} \frac{d_{i} \mu_{j}\left(\left|a_{i j}(t)\right|+\left|b_{i j}(t)\right|\right)}{d_{j} c_{i}(t)}<1 .
$$

We remark that conditions (d) and (4.10) are different and, in [22], they did not consider a similar condition to (4.9) because they assumed that all solutions of (4.8) are defined on $\mathbb{R}$. Thus it is relevant to observe that Corollary 4.4 gives a different stability criterion where it is possible to have unbounded coefficients.

Example 4.3. In [14], the authors studied the global asymptotic stability of the following recurrent neural network model

$$
x_{i}^{\prime}(t)=-c_{i}(t) x_{i}(t)+\sum_{j=1}^{n} \sum_{p=1}^{P} a_{i j p}(t) f_{i j p}\left(\int_{-\infty}^{0} k_{i j p}(-s) x_{j}(t+s) d s\right)+I_{i}(t), t \geq 0, i=1, \ldots, n(4
$$

where $c_{i}, a_{i j p}:[0,+\infty) \rightarrow \mathbb{R}$ are continuous functions, $f_{i j p}: \mathbb{R} \rightarrow \mathbb{R}$ are Lipschitz functions, and the delay kernel functions $k_{i j p}:[0,+\infty) \rightarrow[0,+\infty)$ are piecewise continuous such that

$$
\int_{0}^{\infty} k_{i j p}(s) d s=1 .
$$


System (4.11) arises as another special case of model (4.1), when we consider $\rho_{i}(u)=1, b_{i}(t, u)=$ $c_{i}(t) u, h_{i j p}(u)=0, g_{i j p}(u)=u$ for all $t \geq 0$ and $u \in \mathbb{R}$, and the functions $\eta_{i j p}$ defined by

$$
\eta_{i j p}(s)=\int_{-\infty}^{s} k_{i j p}(-u) d u, \quad s \in(-\infty, 0], i, j=1, \ldots, n, p=1, \ldots, P .
$$

Consequently, from Theorem 4.2, we obtain the following result.

Corollary 4.5. Assume that $f_{i j p}: \mathbb{R} \rightarrow \mathbb{R}$ are $\mu_{\text {ijp }}$-Lipschitz functions.

If there exist $d=\left(d_{1}, \ldots, d_{n}\right)>0$ and $\alpha>0$ such that, for each $i \in\{1, \ldots, n\}$,

$$
d_{i} c_{i}(t)-\sum_{j=1}^{n} \sum_{p=1}^{P} d_{j}\left|a_{i j p}(t)\right| \mu_{i j p}>\alpha \forall t \geq 0, \text { and } \limsup _{t \rightarrow+\infty} \sum_{j=1}^{n} \sum_{p=1}^{P} \frac{d_{j}\left|a_{i j p}(t)\right| \mu_{i j p}}{d_{i} c_{i}(t)}<1,
$$

then the system (4.11) is globally asymptotically stable.

In [14], the authors assumed a different set of hypotheses, also including that $c_{i}(t), a_{i j p}(t)$, and $I_{i}(t)$ are bounded functions, to get the global asymptotic stability of (4.11), since a different norm in $\mathbb{R}^{n}$ was considered.

Example 4.4. Finally, consider the following nonautonomous BAM neural network model with both discrete time-varying delays and distributed delays

$$
\left\{\begin{array}{r}
x_{i}^{\prime}(t)=-\widetilde{b}_{i}\left(t, x_{i}(t)\right)+\sum_{j=1}^{m} a_{i j}(t) f_{i j}\left(\lambda_{j} y_{j}\left(t-\tau_{i j}(t)\right)\right)+\sum_{j=1}^{m} c_{i j}(t) g_{i j}\left(\int_{-\infty}^{0} y_{j}(t+s) d \eta_{i j}(s)\right)+\widetilde{I}_{i}(t) \\
i=1, \ldots, n, \\
y_{j}^{\prime}(t)=-b_{j}\left(t, y_{j}(t)\right)+\sum_{i=1}^{n} \widetilde{a}_{j i}(t) \widetilde{f}_{j i}\left(\widetilde{\lambda}_{i} x_{i}\left(t-\widetilde{\tau}_{j i}(t)\right)\right)+\sum_{i=1}^{n} \widetilde{c}_{j i}(t) \widetilde{g}_{j i}\left(\int_{-\infty}^{0} x_{i}(t+s) d \widetilde{\eta}_{j i}(s)\right)+I_{j}(t) \\
j=1, \ldots, m
\end{array}\right.
$$

where $\lambda_{j}, \widetilde{\lambda}_{i}$ are positive constants. As in the above examples, it is also easy to see that (4.14) is a special case of model (4.1) and, from Theorem 4.2, we obtain the following result.

Corollary 4.6. Assume that $f_{i j}, g_{i j}, \widetilde{f}_{j i}, \widetilde{g}_{j i}: \mathbb{R} \rightarrow \mathbb{R}$ are Lipschitz functions with Lipschitz constants $\mu_{i j}, \sigma_{i j}, \widetilde{\mu}_{j i}, \widetilde{\sigma}_{j i}$ respectively, $b_{j}, \widetilde{b}_{i}:[0+\infty) \times \mathbb{R} \rightarrow \mathbb{R}, a_{i j}, c_{i j}, \widetilde{I}_{i}, \widetilde{a}_{j i}, \widetilde{c}_{j i}, I_{j}:[0,+\infty) \rightarrow \mathbb{R}, \tau_{i j}, \widetilde{\tau}_{j i}:$ $[0,+\infty) \rightarrow[0,+\infty)$ are continuous functions such that $b_{j}$ and $\widetilde{b}_{i}$ satisfy (A2), $t-\tau_{i j}(t) \rightarrow+\infty$, $t-\widetilde{\tau}_{j i}(t) \rightarrow+\infty$ as $t \rightarrow+\infty$, and $\eta_{i j}, \widetilde{\eta}_{j i}:(-\infty, 0] \rightarrow \mathbb{R}$ are non-decreasing, bounded, and normalized functions.

If there exist $d=\left(d_{1}, \ldots, d_{m}\right)>0, \widetilde{d}=\left(\widetilde{d}_{1}, \ldots, \widetilde{d}_{n}\right)>0$, and $\alpha>0$ such that

$$
\begin{aligned}
& \widetilde{d}_{i} \widetilde{\beta}_{i}(t)-\sum_{j=1}^{m} d_{j}\left(\left|a_{i j}(t)\right| \lambda_{j} \mu_{i j}+\left|c_{i j}(t)\right| \sigma_{i j}\right)>\alpha, \quad \forall t \geq 0, i=1, \ldots, n, \\
& d_{j} \beta_{j}(t)-\sum_{i=1}^{n} \widetilde{d}_{i}\left(\left|\widetilde{a}_{j i}(t)\right| \widetilde{\lambda}_{i} \widetilde{\mu}_{j i}+\left|\widetilde{c}_{j i}(t)\right| \widetilde{\sigma}_{j i}\right)>\alpha, \quad \forall t \geq 0, j=1, \ldots, m, \\
& \limsup _{t \rightarrow+\infty} \sum_{j=1}^{m} \frac{d_{j}\left(\left|a_{i j}(t)\right| \lambda_{j} \mu_{i j}+\left|c_{i j}(t)\right| \sigma_{i j}\right)}{\widetilde{d}_{i} \widetilde{\beta}_{i}(t)}<1, \quad i=1, \ldots, n, \\
& \limsup _{t \rightarrow+\infty} \sum_{i=1}^{n} \frac{\widetilde{d}_{i}\left(\left|\widetilde{a}_{j i}(t)\right| \widetilde{\lambda}_{i} \widetilde{\mu}_{j i}+\left|\widetilde{c}_{j i}(t)\right| \widetilde{\sigma}_{j i}\right)}{d_{j} \beta_{j}(t)}<1, \quad j=1, \ldots, m,
\end{aligned}
$$

then the system (4.14) is globally asymptotically stable. 
Now considering (4.14) with $\widetilde{b}_{i}(t, u)=\widetilde{c}_{i}(t) u, b_{j}(t, u)=c_{j}(t) u, f_{i j}(u)=f_{j}(u), \widetilde{f}_{j i}(u)=\widetilde{f}_{i}(u)$, and $c_{i j}(t)=\widetilde{c}_{j i}(t)=0$, then we obtain the following BAM neural network

$$
\left\{\begin{array}{l}
x_{i}^{\prime}(t)=-\widetilde{c}_{i}(t) x_{i}(t)+\sum_{j=1}^{m} a_{i j}(t) f_{j}\left(\lambda_{j} y_{j}\left(t-\tau_{i j}(t)\right)\right)+\widetilde{I}_{i}(t), \quad i=1, \ldots, n, \\
y_{j}^{\prime}(t)=-c_{j}(t) y_{j}(t)+\sum_{i=1}^{n} \widetilde{a}_{j i}(t) \widetilde{f}_{i}\left(\widetilde{\lambda}_{i} x_{i}\left(t-\widetilde{\tau}_{j i}(t)\right)\right)+I_{j}(t), \quad j=1, \ldots, m
\end{array} .\right.
$$

In [25], the authors studied the model (4.19), with periodic coefficients, and they obtained sufficient conditions for the existence of a periodic solution and its global asymptotic stability. In fact they established the following result.

Theorem 4.7. [25] Assume that

(i) the functions $\widetilde{c}_{i}, c_{j}, a_{i j}, \widetilde{a}_{j i}, \widetilde{I}_{i}, I_{j}:[0,+\infty) \rightarrow \mathbb{R}$ are continuously periodic with common period $\omega>0$ and $\tau_{i j}, \widetilde{\tau}_{j i}:[0,+\infty) \rightarrow[0,+\infty)$ are differentiable periodic with the same period $\omega ;$

(ii) the functions $f_{j}, \widetilde{f}_{i}$ are Lipschitz functions with Lipschitz constants $\mu_{j}, \widetilde{\mu}_{i}$ respectively;

(iii) there exist two constants $l_{k}>1(k=1,2)$, with $0<\frac{1}{1-\tau_{i j}^{\prime}(t)}<l_{1}$ and $0<\frac{1}{1-\widetilde{\tau}_{j i}^{\prime}(t)}<l_{2}$, for all $t \geq 0$, such that

$$
\begin{aligned}
& \underline{\widetilde{c}_{i}}-\sum_{j=1}^{m} \overline{a_{i j}} \mu_{j} \lambda_{j} l_{1}>0, \quad i=1, \ldots, n, \\
& \underline{c_{j}}-\sum_{i=1}^{n} \overline{\widetilde{a}_{j i}} \widetilde{\mu}_{i} \widetilde{\lambda}_{i} l_{2}>0, \quad j=1, \ldots, m,
\end{aligned}
$$

where, for a $\omega$-periodic real function $g$, we denote $\bar{g}:=\sup _{t \in[0, \omega]} g(t)$ and $\underline{g}:=\inf _{t \in[0, \omega]} g(t)$.

Then the system (4.19) has a unique $\omega$-periodic solution.

If we consider the model (4.19) with $\omega$-periodic coefficients, then the functions $\widetilde{c}_{i}(t), c_{j}(t), a_{i j}(t)$, and $\widetilde{a}_{j i}(t)$ are bounded, which implies that conditions (4.15) and (4.17) are equivalent to

$$
\widetilde{d}_{i} \widetilde{c}_{i}(t)-\sum_{j=1}^{m} d_{j}\left|a_{i j}(t)\right| \lambda_{j} \mu_{j}>0, \quad \forall t \geq 0, i=1, \ldots, n,
$$

and conditions (4.16) and (4.18) are equivalent to

$$
d_{j} c_{j}(t)-\sum_{i=1}^{n} \widetilde{d}_{i}\left|\widetilde{a}_{j i}(t)\right| \widetilde{\lambda}_{i} \widetilde{\mu}_{i}>0, \quad \forall t \geq 0, j=1, \ldots, m,
$$

for some $d=\left(d_{1}, \ldots, d_{m}\right)>0$ and $\widetilde{d}=\left(\widetilde{d}_{1}, \ldots, \widetilde{d}_{n}\right)>0$. As $l_{k}>1$, the hypothesis (iii) of Theorem 4.7 implies (4.22) and (4.23), thus the following stability criterion is a trivial consequence of Corollary 4.6 and Theorem 4.7 .

Theorem 4.8. Assume conditions (i)-(iii) in Theorem 4.7.

Then the system (4.19) is globally asymptotically stable and it has a unique w-periodic solution.

Remark 4.2. For the $\omega$-periodic BAM neural network model (4.19), Zhang and Zhou [25] proved the existence of a $\omega$-periodic solution and the global asymptotic stability of the model assuming (i), (ii) in Theorem 4.7 and

$$
\begin{aligned}
& \underline{\widetilde{c}_{i}}-\max \left\{\sum_{j=1}^{m} \overline{a_{i j}} \mu_{j} \lambda_{j} l_{1}, \sum_{j=1}^{m} \overline{\widetilde{a}_{j i}} \widetilde{\mu}_{i} \widetilde{\lambda}_{i} l_{2}\right\}>0, \quad i=1, \ldots, n, \\
& \underline{c_{j}}-\max \left\{\sum_{i=1}^{n} \overline{\widetilde{a}_{j i}} \widetilde{\mu}_{i} \widetilde{\lambda}_{i} l_{2}, \sum_{i=1}^{n} \overline{a_{i j}} \mu_{j} \lambda_{j} l_{1}\right\}>0, \quad j=1, \ldots, m,
\end{aligned}
$$


with $l_{k}(k=1,2)$ as in Theorem 4.7.

Since (4.24) implies (4.20), (4.25) implies (4.21), and the conditions are not equivalent, then the Theorem 4.8 improves the main stability result in [25].

\section{Acknowledgments.}

The second author research was suported by the Research Centre of Mathematics of the University of Minho with the Portuguese Funds from the "Fundação para a Ciência e a Tecnologia", through the project PEstOE/MAT/UI0013/2014. The authors thank the referee for valuable comments.

\section{References}

[1] P. Baldi and A. F. Atiya, How delays affect neural dynamics and learning, IEEE Trans. Neural Networks 5 (1994) 612-621.

[2] J. Bélair, Stability in a model of a delayed neural network, J. Dynam. Differential Equations 5 (1993) 607-623.

[3] S. A. Campbell, Delay independent stability for additive neural networks, Differential Equations Dynam. Systems 9 (2001) 115-138.

[4] J. Cao and J. Liang, Boundedness and stability for Cohen-Grossberg neural network with timevarying delays, J. Math. Anal. Appl. 296 (2004) 665-685.

[5] J. Cao and L. Wang, Periodic oscillatory solution of bidirectional associative memory networks with delays, Phys. Rev. E 61 (2) (2000) 1825-1828.

[6] M. Cohen and S. Grossberg, Absolute stability of global pattern formation and parallel memory storage by competitive neural networks, IEEE Trans. Systems Man Cybernet. 13 (1983) 815-826.

[7] S. Esteves, E. Gökmen, and J.J. Oliveira, Global exponential stability of nonautonomous neural network models with continuous distributed delays, Appl. Math. Comput. 219 (2013) 9296-9307.

[8] T. Faria and J.J. Oliveira, General criteria for asymptotic and exponential stabilities of neural network models with unbounded delays, Appl. Math. Comput. 217 (2011) 9646-9658.

[9] J. Haddock and W. Hornor, Precompactness and convergence in norm of positive orbits in a certain fading memory space, Funkcial. Ekvac. 31 (1988) 349-361.

[10] J.K. Hale and J. Kato, Phase Space for Retarded Equations with Infinite Delay, Funkcialaj Ekvacioj, 21 (1978) 11-41.

[11] Y. Hino, S. Murakami, and T. Naito, Functional Differential Equations with Infinite Delay, Springer-Verlag, Berlin, 1991.

[12] J.J. Hopfield, Neural networks with graded response have collective computational properties like those of two-state neurons, Proc. Natl. Acad. Sci. 81 (1984) 3088-3092.

[13] H. Jiang, Z. Li, and Z. Teng, Boundedness and stability for nonautonomous cellular neural networks with delays, Phys. Lett. A 306 (2003) 313-325.

[14] H. Jiang and Z. Teng, Boundedness and global stability for nonautonomous recurrent neural networks with distributed delays, Chaos Solitons \& Fractal 30 (2006) 83-93.

[15] B. Kosko, Bidirectional associative memories, IEEE Trans. Systems Man Cybern. 18 (1988) 49-60.

[16] J. Li, J. Yan, and X. Jia, Dynamical analysis of Cohen-Grossberg neural networks with finite and infinite delays, Appl. Math. Comput. 213 (2009) 529-537. 
[17] B. Liu, New convergence behavior of solutions to shunting inhibitory cellular neural networks with unbounded delays and time-varying coefficients, Appl. Math. Modelling. 33 (2009) 54-60.

[18] C.M. Marcus and R.M. Westervelt, Stability of analogy neural networks with delay, Phys. Rev. A 39 (1989) 347-359.

[19] J.J. Oliveira, Global asymptotic stability for neural network models with distributed delays, Math. Comput. Modelling 50 (2009) 81-91.

[20] J.J. Oliveira, Global stability of a Cohen-Grossberg neural network with both time-varying and continuous distributed delays, Nonlinear Anal. RWA 12 (2011) 2861-2870.

[21] J. Shao, Global exponential convergence for delayed cellular neural networks with a class of general activation functions, Nonlinear Anal. RWA 10 (2009) 1816-1821.

[22] Z. Yuan, L. Yuan, and L. Huang, D. Hu, Boundedness and global convergence of non-autonomous neural networks with variable delays, Nonlinear Anal. RWA 10 (2009) 2195-2206.

[23] R. Wu, Exponential convergence of BAM neural networks with time-varying coefficients and distributed delays, Nonlinear Anal. RWA 11 (2010) 562-573.

[24] Q. Zhang, X. Wei, and J. Xu, Global exponential stability for nonautonomous cellular neural networks with unbounded delays, Chaos Solitons \& Fractal 39 (2009) 1144-1151.

[25] Z. Zhang and Z. Zhou, New conditions on existence and global asymptotic stability of periodic solutions for BAM neural networks with time-varying delays, J. Korean Math. Soc. 48 (2011) $\mathrm{N}^{\mathrm{O}} 2,223-240$.

[26] Q. Zhou and J. Shao, Convergence of Cohen-Grossberg neural networks with delays and timevarying coefficients, Electronic Journal of Differential Equations, Vol. 2008(2008), N73, 1-7. 